\title{
BMJ Open Treatment of tungiasis using a tea tree oil-based gel formulation: protocol for a randomised controlled proof-of- principle trial
}

\author{
Solomon Abrha, ${ }^{1,2}$ Julia K Christenson, ${ }^{1}$ John McEwen, ${ }^{1}$ Wubshet Tesfaye (1) , ${ }^{3}$ \\ Susana Vaz Nery, ${ }^{4}$ Aileen Y Chang, ${ }^{5}$ Tim Spelman, ${ }^{6}$ Sam Kosari, ${ }^{1}$ Gabriel Kigen, ${ }^{7}$ \\ Simon Carroll, ${ }^{1,8}$ Jorg Heukelbach, ${ }^{9}$ Hermann Feldmeier, ${ }^{10}$ \\ Andrew Bartholomaeus, ${ }^{11}$ Mark Daniel, ${ }^{12}$ Gregory M Peterson (D) ,13 \\ Jackson Thomas (i) ${ }^{1}$
}

To cite: Abrha S,

Christenson JK, McEwen J, et al. Treatment of tungiasis using a tea tree oil-based gel formulation: protocol for a randomised controlled proofof-principle trial. BMJ Open 2021;11:e047380. doi:10.1136/ bmjopen-2020-047380

- Prepublication history and additional supplemental material for this paper are available online. To view these files, please visit the journal online (http://dx.doi.org/10.1136/ bmjopen-2020-047380)

Received 15 December 2020 Accepted 05 July 2021

D Check for updates

(c) Author(s) (or their employer(s)) 2021. Re-use permitted under CC BY-NC. No commercial re-use. See rights and permissions. Published by BMJ.

For numbered affiliations see end of article.

Correspondence to Dr Jackson Thomas; Jackson.Thomas@canberra. edu.au

\section{ABSTRACT}

Introduction Tungiasis (sand flea disease or jigger infestation) is a neglected tropical disease caused by penetration of female sand fleas, Tunga penetrans, in the skin. The disease inflicts immense pain and suffering on millions of people, particularly children, in Latin America, the Caribbean and sub-Saharan Africa. Currently, there is no standard treatment for tungiasis, and a simple, safe and effective tungiasis treatment option is required. Tea tree oil (TTO) has long been used as a parasiticidal agent against ectoparasites such as headlice, mites and fleas with proven safety and efficacy data. However, current data are insufficient to warrant a recommendation for its use in tungiasis. This trial aims to generate these data by comparing the safety and efficacy of a $5 \%(\mathrm{v} / \mathrm{w}) \mathrm{TTO}$ proprietary gel formulation with $0.05 \%(\mathrm{w} / \mathrm{v})$ potassium permanganate $\left(\mathrm{KMnO}_{4}\right)$ solution for tungiasis treatment. Methods and analysis This trial is a randomised controlled trial (RCT) in primary schools $(\mathrm{n}=8)$ in SouthWestern Kenya. The study will include school children $(n=88)$ aged $6-15$ years with a confirmed diagnosis of tungiasis. The participants will be randomised in a 1:1 ratio to receive a 3-day two times a day treatment of either $5 \%$ TTO gel or $0.05 \% \mathrm{KMnO}_{4}$ solution. Two viable embedded sandflea lesions per participant will be targeted and the viability of these lesions will be followed throughout the study using a digital handheld microscope. The primary outcome is the proportion of observed viable embedded sand fleas that have lost viability (non-viable lesions) by day 10 (9 days after first treatment). Secondary outcomes include improvement in acute tungiasis morbidities assessed using a validated severity score for tungiasis, safety assessed through adverse events and product acceptability assessed by interviewing the participants to rate the treatment in terms of effectiveness, side effects, convenience, suitability and overall satisfaction.

Ethics and dissemination The trial protocol has been reviewed and approved by the University of Canberra Human Research Ethics Committee (HREC-2019-2114). The findings of the study will be presented at scientific conferences and published in a peer-reviewed journal.
Strengths and limitations of this study

- Given tungiasis affects the most disadvantaged communities, this work has an ultimate objective of reducing the tungiasis burden and improving the health and well-being of affected children and communities in Kenya.

- Educational and community support packages (eg, health education using flip charts) delivered to the participating communities as part of this study, will help facilitate the appropriate control of tungiasis with sustainable benefits to the community.

- If tea tree oil gel effectively treats tungiasis, this would provide compelling evidence for a simple, affordable and effective treatment for tungiasis, which does not require direct supervision by a trained health worker, essentially enabling the communities and/or individuals to manage their own health.

- Compliance with the treatment protocol is likely to be less than ideal in the targeted study settings in Kenya, and we also expect considerable attrition; however, regular follow-up is likely to improve patient compliance and limit attrition.

- The identity of trial interventions could not be concealed to the study participants and caregivers.

Trial registration numbers Australian New Zealand Clinical Trials Registry (ACTRN12619001610123); PACTR202003651095100 and U1111-1243-2294.

\section{INTRODUCTION}

Tungiasis (sand flea disease or jigger infestation), is a neglected tropical disease (NTD) caused by penetration of an ectoparasite, female sand flea, Tunga penetrans. ${ }^{1-3}$ It is rampant in resource-limited communities in Latin America, the Caribbean and subSaharan Africa, ${ }^{2}$ where children (aged 5-14 years) and the elderly ( $\geq 60$ years) are most heavily affected, with prevalence data ranging 
from $50 \%$ to $85 \% .^{4-6}$ No clear estimates of global burden of tungiasis exist, ${ }^{7}$ but according to the WHO, 20 million people are estimated to be at risk of developing tungiasis in South America alone. ${ }^{8}$ Based on Kenyan and Ugandan Ministries of Health, ${ }^{9}{ }^{10}$ about 4 million people suffer from the tungiasis, with another 16 million are at risk. A tungiasis infected person can harbour up to hundreds of parasites, usually on the feet and hands with toes, soles and heels are the sites most frequently affected. ${ }^{6}{ }^{11}$ The infection results in intense inflammation and itching, and frequently occurs with secondary bacterial infections, resulting in abscesses, suppuration, cellulitis, lymphangitis, sepsis, tetanus and post-streptococcal glomerulonephritis. Repeated infection can lead to deformation and loss of nails, and disfigurement of the feet. ${ }^{812-15}$ Tungiasis negatively impacts education (in children), quality of life, household economy and well-being of the affected individuals. ${ }^{8}{ }^{16-19}$

Currently, there is no standard treatment for tungiasis. ${ }^{19}$ Parasiticides such as oral thiabendazole,${ }^{20}$ oral ivermectin $^{21}$ and topical benzyl benzoate ${ }^{22}$ and disinfectants like hydrogen peroxide, ${ }^{9}$ have been explored for tungiasis treatment, but there is little conclusive evidence available on their safety or effectiveness. Our seminal systematic review on this topic ${ }^{23}$ identified eight randomised controlled trials (RCTs) ${ }^{24-31}$ investigated interventions for tungiasis. These included: coconut oil-based lotion (Zanzarin) for prevention; and oral—niridazole and ivermectin; topical-ivermectin lotion, metrifonate lotion, thiabendazole lotion, thiabendazole ointment, dimeticones (NYDA) and a neem seed and coconut oils-based mixture for treatment. Among these, the coconut oilbased lotion for prevention, and dimeticones for treatment of tungiasis displayed the most promise. However, the coconut oil-based lotion is no longer commercially available and dimeticones are expensive and currently not available in tungiasis endemic areas in sub-Saharan Africa including Kenya, thus limiting treatment options to surgical extraction of embedded fleas and bathing feet with $0.05 \%$ potassium permanganate $\left(\mathrm{KMnO}_{4}\right)$ solution. ${ }^{31}{ }^{32}$ In such settings, surgical extraction is frequently performed using unsafe procedures involving sharing of sharp instruments, leading to additional bacterial superinfections and potential transmission of viral pathogens like HIV, hepatitis B and hepatitis C. ${ }^{12} 3334$ Although bathing feet with $0.05 \% \mathrm{KMnO}_{4}$ solution is widely used in Kenya and is recommended by the country's Ministry of Health, ${ }^{9}$ recent trials ${ }^{2531}$ have revealed that it was only marginally effective, killing less than $40 \%$ of embedded fleas.

Thus, there is a critical need for new, safe, effective and affordable treatments for tungiasis. This trial aims to address this unmet critical need by trialling a novel $5 \%$ tea tree oil (TTO) gel-based skin formulation. Unlike current treatment agents used, TTO possesses a unique combination of potent insecticidal, acaricidal, antibacterial, anti-inflammatory and wound healing properties. ${ }^{35} 36$ It has long been used as a helpful topical treatment agent for a variety of epidermal parasitic skin diseases in Australia and Europe, with good safety and efficacy data. ${ }^{37}$ The insecticidal and acaricidal effects of topical formulations of TTO for a range of medical ectoparasites/ pests, including house dust mites, Demodex mites, scabies mites, ticks, headlice and fleas, have been investigated in several in vitro, animal and clinical studies, reporting an efficacy range of $70 \%-100 \%$ against these parasites. ${ }^{38-43}$ TTO is also effective at low concentrations (in vitro) as a bactericide (at 0.002\%-2\%; including against MRSA (methicillin-resistant Staphylococcus aureus)), and as an anti-inflammatory agent $(\leq 0.125 \%) .{ }^{36}$ In sum, an ideal therapeutic candidate for tungiasis should be able to kill the embedded parasite, prevent inflammatory skin reactions and block bacterial infection. The unique parasiticidal, antibacterial and anti-inflammatory properties of TTO appear to hold tremendous potential in reducing the burden of tungiasis and its deadly sequalae. The aim of this RCT is to investigate the safety and efficacy of a $5 \% \mathrm{v} / \mathrm{w}$ TTO-proprietary gel formulation in comparison with the locally endorsed, $0.05 \% \mathrm{w} / \mathrm{v} \mathrm{KMnO}_{4}$ solution for tungiasis treatment in children.

\section{METHODS AND ANALYSIS}

This protocol has been written in line with the Standard Protocol Items: Recommendations for Interventional Trials guidelines (online supplemental file 1). ${ }^{44}$

\section{Study setting and design overview}

The study will be conducted at eight selected primary schools (permission letters obtained from the respective directors of the schools) in Kisii and Nyamira counties, South-Western Kenya where tungiasis is endemic. ${ }^{9}$ Schools have been selected based on the presence of students with tungiasis and willingness of the principals to collaborate in the study. Schools already have strong collaborative working relationships with our community collaborator, Global School Partners (GSP), a local nongovernment and not-for-profit charity organisation in Kenya (GSP). ${ }^{45}$ This pre-existing network of the GSP with school directors and student parents will be used to facilitate the successful completion of this study.

The study is designed as an assessor-blinded, phase II RCT. It will be conducted in the dry season as tungiasis peaks during this period. ${ }^{14}$ Eighty-eight participants with tungiasis will be recruited and randomised in a 1:1 ratio to receive either the $5 \%$ TTO proprietary gel formulation or $0.05 \% \mathrm{KMnO}_{4}$ solution. TTO-gel is a water-based, transparent, skin formulation with excellent spreading properties and pleasing aesthetic characteristics. It contains $5 \% \mathrm{v} / \mathrm{w}$ pure and standard Australian TTO (ISO 4730:2017 and AS 2782:2017), approximately $14 \%$ poloxamer $407 \mathrm{gel}$, and other excipients such as formulation stabilisers, penetration enhancers and preservatives. It will be prepared following the WHO's current Good Manufacturing Practice (Institute of Drug Technology, Australia). $\mathrm{KMnO}_{4}$ solution contains $0.05 \mathrm{~g} \mathrm{KMnO}_{4}$ in 
a litre of water. The selection of $\mathrm{KMnO}_{4}$ solution as the active comparator in this study reflects its status as a local tungiasis treatment used in mass campaigns in children (and adults) in Kenya, ${ }^{46}$ and its being the recommended tungiasis treatment by the Kenyan Ministry of Health. ${ }^{9}$ Study participants' feet will be fully assessed as more than $95 \%$ of embedded sand flea lesions are localised to this site (toes, soles and heels), ${ }^{6} 74$ with lesions staged according to Fortaleza classification system (online supplemental file 2). ${ }^{48}$ The test and control interventions will then be applied two times a day on days 1, 4 and 7 . These treatment days are selected based on the lifecycle of the embedded sand flea. As a sand flea can take up to 1-2 weeks to develop from stage II/III (viable embedded lesions) to stage IV (dying or dead embedded sand flea), ${ }^{28} 48$ the use of the three treatment doses is designed to ensure that any stage II or III embedded sand flea lesion would be killed by the treatments before they die due to their natural course. After the treatment, viability signs of embedded fleas in each participant will be monitored. The proportion of observed viable embedded sand fleas that have lost viability (non-viable lesions) by day 10 will be determined and compared between test and control groups, as the primary outcome.

\section{Study personnel}

The trial will be conducted by a recruitment team and a study team in each school. These teams will be composed of staff members of GSP. ${ }^{45}$ The recruitment team will consist of school nurses led by a recruitment officer. This team will be responsible for liaising with the school directors and caregivers to facilitate the participants' informed consent and allocation procedures. The school directors will be used as mediators to reach out to caregivers and potential participants. The members of the team will receive information and training about the trial particularly the recruitment procedure.

The study team will comprise clinical advisors and clinical assessors, led by one of the clinical advisors. The clinical advisors are experienced medical doctors working in hospitals located in the study areas. The clinical assessors are school nurses who will be responsible for collecting baseline demographic and disease characteristics, treating participants and performing outcome assessments. They will be trained on the overall trial and outcome assessment (viability assessment and staging of the embedded sand fleas), intervention application and safety monitoring procedures. The clinical advisors will supervise the clinical assessors, particularly in outcome assessment procedures, and be consulted in any case of diagnostic uncertainty. The reports of clinical assessors will further be evaluated by a panel of infectious disease specialists or offsite clinical assessors by evaluating the photograph records of each participant.

\section{Sample size calculation}

The sample size calculation is based on the primary outcome measure, assuming the $0.05 \% \mathrm{KMnO}_{4}$ solution will have a $40 \%$ efficacy, ${ }^{25}$ and the $5 \%$ TTO proprietary gel formulation will have a $70 \%$ efficacy at 10 days. There are no reports of clinical trials exploring TTO proprietary gel formulation for tungiasis treatment. Hence, the efficacy of TTO was estimated based on the existing observational studies on tungiasis, the clinical experience of our team members, findings from similar trials exploring other tungiasis treatments and findings of studies (in vitro and in vivo) on TTO against other ectoparasites. To enable the detection of this $30 \%$ difference with at least $80 \%$ power at a significance level of $5 \%$, a sample size of 40 participants per arm (88 in total accounting for $10 \%$ attrition, as seen in similar settings ${ }^{27}$ ) is required.

\section{Study participants}

The study population will consist of school children aged 6-15 years from eight schools with a confirmed diagnosis of tungiasis. The age range of 6-15 years was selected because tungiasis is highly prevalent in this group. ${ }^{49} 50$

\section{Consent and assent}

Before starting the study, face-to-face meetings with caregivers, participants and school directors will be held to explain the objectives of the research and to facilitate an understanding of how the research aligns with community values. The overall procedure of the study, the nature of the disease, the preventive strategies, details of the treatments and risks and benefits of participation will also be explained to caregivers and participants using instruction manuals containing coloured photographic images to ensure they fully understand the consequences of participation. A pictorial consent flipchart will be used and any study documents including information booklet will be translated into the locally spoken language to assist and facilitate the consent process. After this explanation, the participant's legally responsible caregiver/parent will be provided a participant information sheet and asked to complete an informed consent with written assent (if aged 12-15 years) or verbal assent (if aged 6-11 years) provided by children (online supplemental files 3 and 4). If a subject and his/her caregiver are unable to read, an impartial witness must be present during the entire informed consent discussion. The signature of the impartial witness will certify the subject's consent. The participant's parent/caregiver will receive a signed and dated copy of the consent form.

\section{Recruitment and enrolment}

Potential participants with tungiasis will be identified in each school and recruited by the recruitment team over 3 months. Eligibility assessment (presence of viable embedded sand fleas) will be initiated by the clinical assessors under the supervision of the clinical advisor as per the inclusion and exclusion criteria. If a potential participant meets the study criteria, he or she will be invited to a room designated for study procedures, referred to as a study centre, for further examination. 
Participants' must have at least one viable embedded sand flea lesion (stage II or stage III) as inclusion criterium. Viable embedded sand flea lesions located at the tip of toes, soles and rim of heels will be exclusively selected for this purpose.

Participants' exclusion criteria are (1) participants with cluster lesions ( $\geq 3$ together) or manipulated lesions; (2) the presence of complicated sand flea lesions requiring antibiotic treatment (these children will be referred to nearby health facilities for appropriate management); (3) evidence that guardian/parent/caregiver intend to change their place of residence during the study period; (4) known history of allergy to any of the study medications (TTO or other essential oils and/or $\mathrm{KMnO}_{4}$ ) and (5) the use of systemic or topical drugs or medications, particularly antibiotics, which may interfere with the study results.

Eligible participants will be instructed to come back to the study centre located in each school for randomisation, baseline assessment, treatment and outcome assessments. An outline of the recruitment and enrolment process with study timeline is given in figure 1 .

\section{Randomisation and treatment allocation}

Participants will be allocated to either the test (5\% TTO gel) or control $\left(0.05 \% \mathrm{KMnO}_{4}\right.$ solution) group in a $1: 1$ ratio using a predetermined, computer-generated randomisation schedule developed by an independent statistician who will not be directly involved in the study. All participants in each school will be allocated in the study with participant from new schools included to the study until the minimum sample is reached. The randomisation schedule will be kept secure (password-protected) by the statistician. The randomisation schedule will be concealed from trial participants, clinical assessors and data assessors (who will be analysing the data) until the participants have been assigned into the trial.

\section{Blinding}

Foot bathing with the $\mathrm{KMnO}_{4}$ solution may change the colour of the skin to dark purple. As a result, the trial participants and onsite clinical assessors cannot be blinded to the trial interventions. However, a blind assessment of photographs of tungiasis lesions by an expert panel of clinicians (offsite clinical assessors) during the data analysis phase will prevent any likelihood of investigator bias in the outcome assessments. To keep the offsite clinical assessors and data assessors blind, they will not be involved in the clinical trial procedures or have any contact with trial participants. Given the primary efficacy outcome will be measured 3 days after the last treatment, we do not consider that the colour of $\mathrm{KMnO}_{4}$ solution on the feet would compromise the blinding of offsite clinical assessors. The onsite team will carefully assess the skin surrounding the targeted lesions and ensure the absence of any trace of purple staining prior to taking the photographs. In any case that the blinding is broken, the study team will document the date and reason for breaking.

\section{Study participant treatment}

The randomised participants will receive either the test (5\% TTO-proprietary gel formulation) or control $(0.05 \%$ $\mathrm{KMnO}_{4}$ solution) intervention. They will be required to attend the study centre in each school two times a day (AM and PM) on days 1, 4 and 7 for the treatments. At each visit the feet of the participants will be washed with water and soap, dried with a clean towel and toenails clipped as necessary to enable the easier application of the products. The allocated treatments will be applied by the clinical assessors. The test product will be applied by smearing the required amount (up to $8 \mathrm{~g} /$ day) of the product on the palms and spreading it over the skin surface of the feet up to the ankle including the soles, and interdigital areas (between toes). The treated feet will then be left for about $15 \mathrm{~min}$ to allow the test products to dry. In contrast, the comparator product will be applied by immersing and bathing the feet up to the ankle in a bucket containing a $0.05 \% \mathrm{KMnO}_{4}$ solution (up to 2.5 L) for $15 \mathrm{~min}$. After sun drying the feet, a thin layer of petroleum jelly, fully covering the treated surface, will be applied for the purpose of softening the roughness on the skin caused by the $\mathrm{KMnO}_{4}$ treatment.

After the initial treatment (day $1 \mathrm{AM}$ ), all participants will be given pairs of closed shoes to be worn throughout the study period and to be kept after the study participation. This will help the treatments remain on the feet and protect the feet from contamination with dirt and water. Also, wearing closed shoes may decrease reinfestation. Participants will be advised to avoid using or mixing any other tungiasis treatments with trial medications during the study period. They will also be advised at each visit to regularly wear the provided pair of shoes throughout the study period. Dates and times of start and end of treatment application, as well as any non-compliance with the trial protocol will be documented in the case report form (CRF, online supplemental file 5).

\section{Outcome assessment}

\section{Primary outcome}

The primary and secondary efficacy end points are the proportion of observed viable embedded sand fleas that have lost viability (non-viable lesions) by day 10 (9 days after first treatment) and by day 5 (4 days after the first treatment), respectively. Participants will be required to attend the study centre in each school once daily (AM or PM) at baseline, days 5 and 10 for the outcome assessment. At baseline, viability of the embedded sand flea lesions located in the feet will be assessed using a handheld digital video microscope, assisted with pictorial flipcharts. Sites of all viable (stage II-III) lesions will be recorded on the foot diagram sheets and the entire feet and appearance will be photographed to document the baseline characteristics of the embedded sandflea lesions. Two viable embedded sand flea lesions will be selected as target lesions and will then be observed for their viability at each outcome assessment visit. All the information collected at baseline, such as the number of viable embedded sand flea lesions, 
- Recruitment process initiated by recruitment team through identification of school children with tungiasis in the selected schools

- Caregivers provided with trial information (Participant Information Sheet)

- Caregiver asked for informed consent

- Child asked for written/verbal assent

- Eligibility assessment by Clinical assessors

- Tungiasis diagnosis confirmed ( $\geq 1$ embedded sand flea in the feet) by the clinical assessors

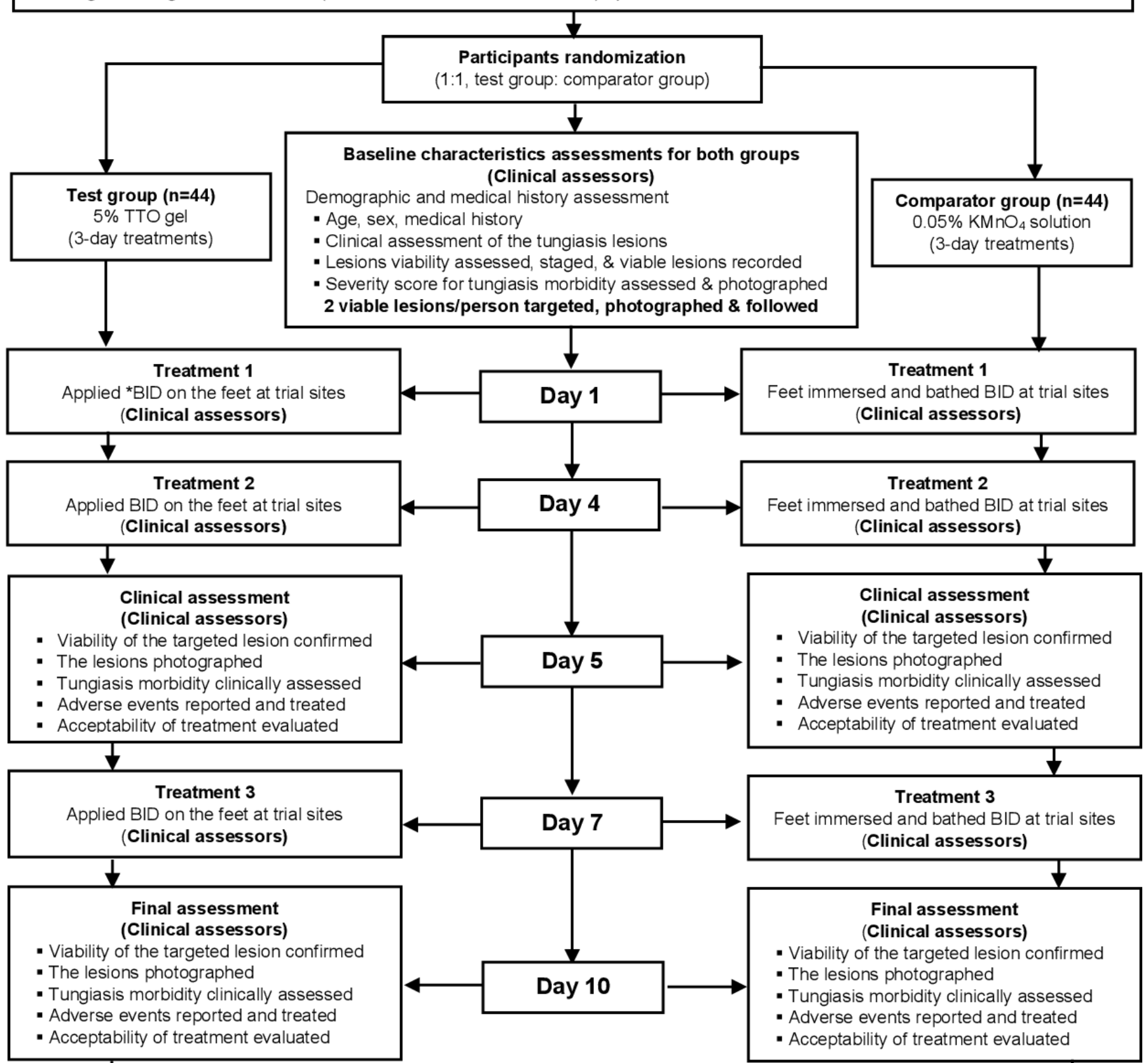

- Viability of the targeted lesion confirmed

- The lesions photographed

- Tungiasis morbidity clinically assessed

- Acceptability of treatment evaluated

Participa

:1, test group: comparator group)

(Clinical assessors)

(Clical assessment

- Clinical assessment of the tungiasis lesions

Severity score for tungiasis morbidity assessed \& photographe 
interventions will be recorded for each study participant at each follow-up visit. Photographs will be recorded and reviewed during the analysis phase to confirm observations recorded in the CRF.

A panel of blinded offsite clinical assessors will evaluate photographs of the targeted embedded sand flea lesions taken at baseline, days 5 and 10 independently of the onsite clinical assessors and the primary outcome measure will be determined by the blinded photograph assessment of the offsite clinical assessor. Any discrepancy in the assessment results will be adjudicated by a third person. An empirical evaluation of the onsite versus offsite agreement will be performed using the kappa coefficient to determine reliability of the assessment. To evaluate the efficacy of the test intervention, the proportions of nonviable lesions in the test group will be compared with the control groups at day 10.

\section{Secondary outcomes}

The secondary outcomes are SSAT, itching, pain, painrelated and itching-related sleep disturbance, safety and participant acceptability of the trial intervention/s. The SSAT, which includes typical signs of local inflammation (erythema, oedema and warmness) and the presence of suppuration, ulcers and fissures, will be evaluated by the clinical assessors at baseline, days 5 and 10, using a validated scoring system designed for tungiasis morbidity assessment. ${ }^{51}$ The entire feet and appearance will be photographed and recorded in the CRF to evaluate this outcome measure. The itch-man scale for itching, ${ }^{52}$ and 4-point tungiasis pictorial scales ${ }^{18}$ for pain, and pain and itching-related sleep disturbance will be used to evaluate these outcomes.

Safety will be assessed through adverse events (AEs) and evaluations of the skin irritation during each visit (days 1, 4, 5, 7 and 10). Participants/caregivers (in-person or on the phone) will be asked at each follow-up visit by the study team about the occurrence of local (stinging/ burning, irritation and itching) or systemic AEs (nausea and headache). Children will be physically examined for evidence of local swelling, erythema and fever. The severity of the AEs will be categorised as mild (grade 1), moderate (grade 2), severe (grade 3 ) and life-threatening (grade 4) according to the Common Terminology Criteria for Adverse Events (CTCAE) version 5.0 guideline (online supplemental file 2). ${ }^{53}$ Acceptability of the treatments will be assessed at the end of the study (day 10) by asking the participants to rate the treatment in terms of effectiveness, side effects, convenience, suitability and overall satisfaction. Responses to these questions will be recorded in the CRF.

\section{Adherence and retention}

Continuous motivation and advice will be given by the clinical assessors to the participants at each visit throughout the study to promote study retention. Participants and/ or carers will also be asked to complete a treatment diary recording the daily progression of the condition-which will reinforce the need for optimum treatment compliance. Community home visits will be organised, if required (eg, in case of absenteeism from school).

\section{Monitoring and reporting of AEs}

If AEs occur, the clinical advisors will determine the relationship between the AEs and the trial medication. AEs considered related to the trial medication will be followed up until either resolution, or the event is considered stable. All grade 1 and 2 AEs reported spontaneously by the subject or observed by the study team will be recorded in the $\mathrm{AE}$ form and documented in each participant's CRF. The following information about each AE will also be recorded where available: description, onset and end date, severity, expectedness, assessment of relatedness to trial medication, what action was taken afterwards and whether the participant was withdrawn from the trial.

A serious AEs (SAEs) will also be reported to the Human Ethics Committees and regulatory bodies as per the reporting schedule stipulated in their guidelines. The following information will be documented in the SAE form: description, classification, start date, status/ outcome, relatedness to study intervention, therapy given and any actions taken to study intervention.

\section{Statistical analysis}

All data will be reported following the Consolidated Standards of Reporting Trials guidelines (online supplemental file 6) ${ }^{54}$ A detailed analysis plan will be approved by all investigators before any data analysis. The data will be analysed by the study statistician who will be blinded to the treatment allocation. Statistical analyses will be performed for both the intention to treat (ITT) and per-protocol (PP) populations. The ITT population will include all randomised participants treated or not, and any participants who withdraw prematurely or poorly comply with the protocol. The PP population will be all subjects who are enrolled in the study, randomly assigned to the treatment regimen, received three doses (two times a day) and do not deviate from the study protocol in a clinically significant manner. Results will be considered significant if $\mathrm{p} \leq 0.05$.

Baseline characteristics collected on each patient will be reported and compared between randomisation groups including age, sex, number of viable embedded sand flea lesions, SSAT, as well as scores for pain, itching and sleep disturbance. Categorical (qualitative) variables will be summarised by frequency and percentage. Continuous variables will be summarised as mean and SD in case of normal distribution and as median and IQR in case of non-normal distribution. The Shapiro-Wilk test will be used to assess the normality of the distribution of outcome variables for both groups. Independent Student's t-test or Mann-Whitney tests will be used to investigate differences in continuous variables, and $\chi^{2}$ tests will be used to identify significant variations in proportions across treatment groups. 
Based on the change in primary outcome, the efficacy of test and comparator products will be compared at each follow-up visit. The difference in proportion of nonviable lesions between the test and control groups will be compared using Student's t-test or Mann-Whitney tests depending on the distribution and presented as relative and absolute risk reductions with their respective 95\% CIs and $p$ values. Further, within-group differences will be assessed using paired t-test in case of normally distributed data and a Wilcoxon signed-rank test in case of nonnormally distributed data. Secondary outcomes will be compared in the same fashion as the primary outcome.

\section{Study management}

Quality assurance audits of the clinical trial and related documentation will be performed during and after this study in accordance with the International Conference on Harmonisation Good Clinical Practice (ICH-GCP) guidelines and recommendations. ${ }^{55} 56$ The quality assurance will also consider the Kenyan GCP guideline and the Pharmacy and Poisons Board (PPB) requirements. Trial standard operating procedures (SOPs) will be used to ensure that the trial will be conducted, and data are generated, documented (recorded) and reported in compliance with the latest approved protocol, ICHGCP, Declaration of Helsinki, Kenyan GCP, PPB and National Commission for Science Technology and Innovation (NACOSTI) requirements. The data monitoring committee will involve a medical practitioner, toxicologist and pharmacist.

\section{Patient and public involvement}

Patients and/or the public will not involve in the study design, conduct, reporting or dissemination plans of this research. Study results and feedback will be disseminated to patients by local trial team in formats that are useful and understandable, such as community meetings, presentations, websites and social media.

\section{ETHICS AND DISSEMINATION \\ Ethical approval}

The trial protocol has been approved on 29 August 2019 by the University of Canberra Human Research Ethics Committee (HREC20192114) and registered with WHO accredited registries (online supplemental files 7-9). Further, the investigators will secure ethical approvals from one of the NACOSTI (Kenya) accredited ethics review committees and will seek letters of support from both the Kenyan Ministry of Health and Ministry of Education.

\section{Confidentiality and access to data}

The privacy of participants will be protected by appropriate collection and storage of data. Participants will be identified only by initials and participant ID numbers on the CRFs and in any electronic databases. Data collection forms will be stored in locked filing cabinets in a locked office at the participating schools until the end of the study period, which will then be transferred to the University of Canberra and handled as per the university's recommended data storage guideline for clinical trials. All documents will only be accessible by trial staff and authorised personnel. Documents containing participant's identifying information will not be stored electronically and will be anonymised as soon as practical. Participants will be advised their records may be examined by lawful authorities but will be treated with strict confidentiality and will not be made publicly available.

\section{Dissemination}

Study results and feedback will be disseminated to endusers (participants and community members, counties' health bureaus and other relevant government organisations) in formats that are useful and understandable, such as community meetings, presentations, websites, social media and radio announcements. The findings of this study will also be disseminated through peerreviewed journals and national and international scientific meetings.

\section{DISCUSSION}

In endemic communities, tungiasis morbidity is caused by the parasite and associated inflammatory skin reactions and secondary bacterial infections. Thus, proposed treatment options should have the potential to address the morbidities caused by the parasite and treat secondary bacterial complications. In this vein, TTO is a strong fit for tungiasis treatment as it possesses a unique combination of parasiticidal, antibacterial, anti-inflammatory and wound healing properties. ${ }^{36}$ There has been a claim that TTO causes skin irritation or allergic contact dermatitis. ${ }^{57}$ In a suitable pharmaceutical base at concentrations $\leq 25 \%$, multiple clinical studies $^{58-64}$ have shown that TTO has no or low risk of adverse skin reactions. While potential toxicity in children is yet to be extensively evaluated, a report from an $\mathrm{RCT}^{65}$ in children (mean age $6.3+5.1$ years) with viral molluscum contagiosum demonstrated that $75 \%(\mathrm{v} / \mathrm{v})$ TTO was well tolerated in the 30-day treatment period. TTO's sensitising potential is largely due to elevated levels of peroxides and other degradation products from oxidised oil. ${ }^{66}$ When correctly stored in amber glass bottles with polypropylene caps, TTO has no appreciable degradation for up to 12 months. ${ }^{3657}$ Due to its high volatility, $90 \%$ of the applied TTO rapidly evaporates, minimising the potential for components to permeate the dermis and bloodstream. Nevertheless, key active components (terpinen-4-ol, $\alpha$-terpineol and 1,8-cineole) have sufficient epidermal penetration to provide antimicrobial, anti-inflammatory and potentially insecticidal and acaricidal effects. ${ }^{35}$

If TTO gel effectively treats tungiasis, this trial will provide compelling evidence for a simple, affordable and 
effective treatment for disadvantaged populations with a significant health burden. This will lead to a significant change in the treatment of this neglected condition. While the tungiasis-affected children in selected Kenyan villages are intended as the primary beneficiaries of this research, the pattern of tungiasis and associated bacterial complications among children is analogous to that observed in resource-poor and underprivileged endemic communities in many parts of the world, especially in subSaharan Africa. Thus, the results from this study have the potential to provide evidence for a global health role of TTO in managing tungiasis and its associated complications in children.

\section{Author affiliations}

${ }^{1}$ Pharmacy, Faculty of Health, University of Canberra, Canberra, Australian Capital Territory, Australia

${ }^{2}$ Department of Pharmaceutics, Mekelle University, Mekelle, Tigray, Ethiopia

${ }^{3}$ Pharmacy, Health Research Institute, University of Canberra, Canberra, Australian Capital Territory, Australia

${ }^{4}$ The Public Health Interventions Research Program, The Kirby Institute for Infection and Immunity in Society, University of New South Wales, Sydney, New South Wales, Australia

${ }^{5}$ Department of Dermatology, University of California San Francisco, San Francisco, California, USA

${ }^{6}$ Public Health, Burnet Institute International Health Research Group, Melbourne, Victoria, Australia

${ }^{7}$ Department of Pharmacology and Toxicology, Moi University School of Medicine, Eldoret, Central, Kenya

${ }^{8}$ Global School Partners, Canberra, Australian Capital Territory, Australia

${ }^{9}$ Department of Community Health, Federal University of Ceará, Fortaleza, Brazil, Fortaleza, Brazil

${ }^{10}$ Institute of Microbiology and Infection Immunology, Campus Benjamin Franklin, Charité University Medicine, Berlin, Germany

${ }^{11}$ Diamantina Institute, The University of Queensland, Woolloongabba, Queensland, Australia

${ }^{12}$ Centre for Research and Action in Public Health, Health Research Institute, University of Canberra, Canberra, Australian Capital Territory, Australia

${ }^{13}$ School of Pharmacy, University of Tasmania, Hobart, Tasmania, Australia

Contributors SA and JT conceived the study. GMP, AB, JKC, JH, JM and SC contributed to the study design. SA and JT drafted the manuscript. JKC, JM, WT, SVN, AC TS, SK, GK, SC, JH, HF, AB, MD and GMP assisted in developing the protocol and have reviewed and edited the manuscript. All authors have read and approved the final manuscript.

Funding The authors have not declared a specific grant for this research from any funding agency in the public, commercial or not-for-profit sectors.

Competing interests None declared.

Patient consent for publication Not required.

Provenance and peer review Not commissioned; externally peer reviewed.

Supplemental material This content has been supplied by the author(s). It has not been vetted by BMJ Publishing Group Limited (BMJ) and may not have been peer-reviewed. Any opinions or recommendations discussed are solely those of the author(s) and are not endorsed by BMJ. BMJ disclaims all liability and responsibility arising from any reliance placed on the content. Where the content includes any translated material, BMJ does not warrant the accuracy and reliability of the translations (including but not limited to local regulations, clinical guidelines, terminology, drug names and drug dosages), and is not responsible for any error and/or omissions arising from translation and adaptation or otherwise.

Open access This is an open access article distributed in accordance with the Creative Commons Attribution Non Commercial (CC BY-NC 4.0) license, which permits others to distribute, remix, adapt, build upon this work non-commercially, and license their derivative works on different terms, provided the original work is properly cited, appropriate credit is given, any changes made indicated, and the use is non-commercial. See: http://creativecommons.org/licenses/by-nc/4.0/.
ORCID iDs

Wubshet Tesfaye http://orcid.org/0000-0001-7208-2330

Gregory M Peterson http://orcid.org/0000-0002-6764-3882

Jackson Thomas http://orcid.org/0000-0002-0699-788X

\section{REFERENCES}

$1 \mathrm{WHO}$ /Department of control of neglected tropical diseases. Recognizing neglected tropical diseases through changes on the skin: A training guide for front-line health workers [internet]. Geneva, Swizerland: WHO; 2018 [cited 2018 June 1]. Available: http:// www.who.int/neglected_diseases/resources/9789241513531/en/ [Accessed 9 Oct 2018].

2 Heukelbach J, de Oliveira FAS, Hesse G, et al. Tungiasis: a neglected health problem of poor communities. Trop Med Int Health 2001;6:267-72.

3 Miller H, Trujillo-Trujillo J, Mutebi F, et al. Efficacy and safety of dimeticones in the treatment of epidermal parasitic skin diseases with special emphasis on tungiasis: an evidence-based critical review. Braz J Infect Dis 2020;24:170-7.

4 Muehlen M, Heukelbach J, Wilcke T, et al. Investigations on the biology, epidemiology, pathology and control of Tunga penetrans in Brazil. II. prevalence, parasite load and topographic distribution of lesions in the population of a traditional fishing village. Parasitol Res 2003:90:449-55.

5 Wilcke T, Heukelbach J, César Sabóia Moura R, et al. High prevalence of tungiasis in a poor neighbourhood in Fortaleza, northeast Brazil. Acta Trop 2002;83:255-8.

6 Ugbomoiko US, Ofoezie IE, Heukelbach J. Tungiasis: high prevalence, parasite load, and morbidity in a rural community in Lagos state, Nigeria. Int J Dermatol 2007;46:475-81.

7 Feldmeier H, Eisele M, Van Marck E, et al. Investigations on the biology, epidemiology, pathology and control of Tunga penetrans in Brazil: IV. Clinical and histopathology. Parasitol Res 2004;94:275-82.

8 WHO. Scabies and other ectoparasites [internet]. Geneva, Switzerland: WHO, 2020. Available: https://www.who.int/neglected diseases/diseases/scabies-and-other-ectoparasites/en/index1.html [Accessed 6 Feb 2020].

9 Ministry of Health. National policy guidelines on prevention and control of jigger infestations [internet]. Nairobi, Kenya: Division of Environmental Health; 2014 [cited 2014 January 1]. Available: http:// guidelines.health.go.ke/\#/category/12/95/meta [Accessed 3 Oct 2018].

10 Ministry of Health. Press statement on new master plan to tackle targeted neglected tropical diseases [internet]. Uganda: Republic of Uganda Ministry of Health, 2012. Available: http://www.health.go. ug/docs/Press\%20statement\%20on\%20NTDs.pdf [Accessed 2 Oct 2018].

11 Feldmeier H, Eisele M, Sabóia-Moura RC, et al. Severe tungiasis in underprivileged communities: case series from Brazil. Emerg Infect Dis 2003:9:949-55.

12 Feldmeier H, Sentongo E, Krantz I. Tungiasis (sand flea disease): a parasitic disease with particular challenges for public health. Eur $J$ Clin Microbiol Infect Dis 2013;32:19-26.

13 Feldmeier $\mathrm{H}$, Heukelbach J, Eisele M, et al. Bacterial superinfection in human tungiasis. Trop Med Int Health 2002;7:559-64.

14 Nyangacha RM, Odongo D, Oyieke F, et al. Secondary bacterial infections and antibiotic resistance among tungiasis patients in Western, Kenya. PLoS Negl Trop Dis 2017;11:e0005901.

15 Veraldi S, Valsecchi M. Imported tungiasis: a report of 19 cases and review of the literature. Int $J$ Dermatol 2007;46:1061-6.

16 Josephine N, Peter NK, Walter M. Impact of tungiasis on acquisition of basic education among children aged 5-14 years in Murang'a County, Kenya. Int J Innov Res Sci Eng Technol 2015;2:128-42.

17 Josephine N, NK P, Walter M. Quantifying burden of disease caused by tungiasis using disability adjusted life years metric among the children aged 5-14 years in Murang' a County, Kenya. Int $J$ Environ Res Public Health 2015;2:151-8.

18 Wiese S, Elson L, Feldmeier $\mathrm{H}$. Tungiasis-related life quality impairment in children living in rural Kenya. PLoS Negl Trop Dis 2018;12:e0005939.

19 Elson L, Fillinger U, Feldmeier H. Tungiasis. In: Tyring SK, Lupi O, Hengge UR, eds. Tropical dermatology. 2nd edn. UK: Elsevier BV, 2017: 401-4.

20 Cardoso A. Generalized Tungiasis treated with thiabendazole. Arch Dermatol 1981;117:127.

21 Heukelbach J, Wilcke T, Winter B, et al. Efficacy of ivermectin in a patient population concomitantly infected with intestinal helminths and ectoparasites. Arzneimittelforschung 2004;54:416-21. 
22 Mitchell CJ, Stephany P. Infestation of Tunga penetrans in villages near Zomba Central Hospital. Malawi Med J 2013;25:88-9.

23 Abrha S, Heukelbach J, Peterson GM, et al. Clinical interventions for tungiasis (sand flea disease): a systematic review. Lancet Infect Dis 2021. doi:10.1016/S1473-3099(20)30853-7. [Epub ahead of print: 05 Jul 2021] https://doi.org/10.1016/S1473-3099(20)30853-7

24 Ade-Serrano MA, Olomolehin OG, Adewunmi A. Treatment of human tungiasis with niridazole (ambilhar) a double-blind placebo-controlled trial. Ann Trop Med Parasitol 1982;76:89-92.

25 Thielecke M, Nordin P, Ngomi N, et al. Treatment of Tungiasis with dimeticone: a proof-of-principle study in rural Kenya. PLoS Negl Trop Dis 2014;8:e3058.

26 Nordin P, Thielecke M, Ngomi N, et al. Treatment of tungiasis with a two-component dimeticone: a comparison between moistening the whole foot and directly targeting the embedded sand fleas. Trop Med Health 2017;45:6.

27 Heukelbach J, Franck S, Feldmeier $\mathrm{H}$. Therapy of tungiasis: a double-blinded randomized controlled trial with oral ivermectin. Mem Inst Oswaldo Cruz 2004;99:873-6.

28 Heukelbach J, Eisele M, Jackson A, et al. Topical treatment of tungiasis: a randomized, controlled trial. Ann Trop Med Parasitol 2003;97:743-9.

29 Buckendahl J, Heukelbach J, Ariza L, et al. Control of tungiasis through intermittent application of a plant-based repellent: an intervention study in a resource-poor community in Brazil. PLoS Negl Trop Dis 2010;4:e879.

30 Thielecke M, Raharimanga V, Rogier C, et al. Prevention of tungiasis and tungiasis-associated morbidity using the plant-based repellent Zanzarin: a randomized, controlled field study in rural Madagascar. PLoS Negl Trop Dis 2013;7:e2426.

31 Elson L, Randu K, Feldmeier $\mathrm{H}$, et al. Efficacy of a mixture of neem seed oil (Azadirachta indica) and coconut oil (Cocos nucifera) for topical treatment of tungiasis. A randomized controlled, proof-ofprinciple study. PLoS Negl Trop Dis 2019;13:e0007822-e22.

32 Elson L, Wright K, Swift J, et al. Control of Tungiasis in absence of a roadmap: Grassroots and global approaches. Trop Med Infect Dis 2017;2:33.

33 Kamau T, House SK. The potential risk of HIV infection and transmission of other blood-borne pathogens through the sharing of needles and pins among people infested with jiggers in Kenya. Int $J$ Med Sci Public Health 2014;4:278-85.

34 Winter B, Oliveira FA, Wilcke T, et al. Tungiasis-related knowledge and treatment practices in two endemic communities in northeast Brazil. J Infect Dev Ctries 2009;3:458-66.

35 Carson CF, Hammer KA, Riley TV. Melaleuca alternifolia (tea tree) oil: a review of antimicrobial and other medicinal properties. Clin Microbiol Rev 2006;19:50-62.

36 Thomas J, Carson CF, Peterson GM, et al. Therapeutic potential of tea tree oil for scabies. Am J Trop Med Hyg 2016;94:258-66.

37 Lauten JD, Boyd L, Hanson MB, et al. A clinical study: Melaleuca, manuka, Calendula and green tea mouth rinse. Phytother Res 2005;19:951-7.

38 Jandourek A, Vaishampayan JK, Vazquez JA. Efficacy of Melaleuca oral solution for the treatment of fluconazole refractory oral candidiasis in AIDS patients. AIDS 1998;12:1033-7.

39 Pazyar N, Yaghoobi R, Bagherani N, et al. A review of applications of tea tree oil in dermatology. Int J Dermatol 2013;52:784-90.

40 Callander JT, James PJ. Insecticidal and repellent effects of tea tree (Melaleuca alternifolia) oil against Lucilia cuprina. Vet Parasitol 2012;184:271-8.

41 Yim WT, Bhandari B, Jackson L, et al. Repellent effects of Melaleuca alternifolia (tea tree) oil against cattle tick larvae (Rhipicephalus australis) when formulated as emulsions and in $\beta$-cyclodextrin inclusion complexes. Vet Parasitol 2016;225:99-103.

42 Walton SF, McKinnon M, Pizzutto S, et al. Acaricidal activity of Melaleuca alternifolia (tea tree) oil: in vitro sensitivity of Sarcoptes scabiei var hominis to terpinen-4-ol. Arch Dermatol 2004;140:563-6.

43 Murphy O, O'Dwyer V, Lloyd-McKernan A. The efficacy of tea tree face wash, 1, 2-Octanediol and microblepharoexfoliation in treating Demodex folliculorum blepharitis. Cont Lens Anterior Eye 2018;41:77-82.
44 Chan A-W, Tetzlaff JM, Altman DG, et al. Spirit 2013 statement: defining standard protocol items for clinical trials. Ann Intern Med 2013;158:200-7.

45 Global School Parteners. GSP-helping the world meet the sustainable development goals: Global School Parteners; 2019 [updated June, 2018]. Available: https://www.globalschoolpartners. org.au [Accessed 26 Feb 2019].

46 Ahadi Kenya Trust. The jigger menace in Kenya report [internet] Nairobi, Kenya: Ahadi Kenya Trust; 2011 [Vol. 2]. Available: http:// www.jigger-ahadi.org/index.html [Accessed 2 Oct 2018].

47 Heukelbach J, Wilcke T, Eisele M, et al. Ectopic localization of tungiasis. Am J Trop Med Hyg 2002;67:214-6.

48 Eisele M, Heukelbach J, Van Marck E, et al. Investigations on the biology, epidemiology, pathology and control of Tunga penetrans in Brazil: I. Natural history of tungiasis in man. Parasitol Res 2003;90:87-99.

49 Girma M, Astatkie A, Asnake S. Prevalence and risk factors of tungiasis among children of Wensho district, southern Ethiopia. BMC Infect Dis 2018;18:456.

50 Dassoni F, Polloni I, Margwe SB, et al. Tungiasis in northern Tanzania: a clinical report from Qameyu village, Babati district, Manyara region. $J$ Infect Dev Ctries 2014;8:1456-60.

51 Kehr JD, Heukelbach J, Mehlhorn $\mathrm{H}$, et al. Morbidity assessment in sand flea disease (tungiasis). Parasitol Res 2007;100:413-21.

52 Morris V, Murphy LM, Rosenberg M, et al. Itch assessment scale for the pediatric burn survivor. J Burn Care Res 2012;33:419-24.

53 US Department of Health and Human Services. Common terminology criteria for adverse events (CTCAE). In: National Institutes of health $\mathrm{NCl}$. USA: National Institutes of Health, National Cancer Institute, 2017.

54 Moher D, Hopewell S, Schulz KF, et al. Consort 2010 explanation and elaboration: updated guidelines for reporting parallel group randomised trials. J Clin Epidemiol 2010;63:e1-37.

55 Dixon JR. The International Conference on harmonization good clinical practice guideline. Quality Assurance 1999;6:65-74.

56 International Council for Harmonisation of Technical Requirements for Pharmaceuticals for Human Use (ICH). ICH harmonised guideline: integrated addendum to ICH E6(R1): Guideline for good clinical practice E6(R2) [internet]. Geneva, Switzerland: ICH, 2016. Available: https://www.ich.org/page/efficacy-guidelines [Accessed 19 Feb 2020].

57 Hammer KA, Carson CF, Riley TV, et al. A review of the toxicity of Melaleuca alternifolia (tea tree) oil. Food Chem Toxicol 2006;44:616-25.

58 Caelli M, Porteous J, Carson CF, et al. Tea tree oil as an alternative topical decolonization agent for methicillin-resistant Staphylococcus aureus. J Hosp Infect 2000;46:236-7.

59 Dryden MS, Dailly S, Crouch M. A randomized, controlled trial of tea tree topical preparations versus a standard topical regimen for the clearance of MRSA colonization. J Hosp Infect 2004;56:283-6.

60 Enshaieh S, Jooya A, Siadat AH, et al. The efficacy of 5\% topical tea tree oil gel in mild to moderate acne vulgaris: a randomized, doubleblind placebo-controlled study. Indian J Dermatol Venereol Leprol 2007;73:22-5.

61 Satchell AC, Saurajen A, Bell C, et al. Treatment of dandruff with 5\% tea tree oil shampoo. J Am Acad Dermatol 2002;47:852-5.

62 Syed TA, Qureshi ZA, Ali SM, et al. Treatment of toenail onychomycosis with $2 \%$ butenafine and $5 \%$ Melaleuca alternifolia (tea tree) oil in cream. Trop Med Int Health 1999;4:284-7.

63 Tong MM, Altman PM, Barnetson RS. Tea tree oil in the treatment of tinea pedis. Australas J Dermatol 1992;33:145-9.

64 RLP L, Leung PHM, Wong TKS. A randomized controlled trial of topical tea tree preparation for MRSA colonized wounds. Int J Nurs Sci 2014:1:7-14.

65 Markum E, Baillie J. Combination of essential oil of Melaleuca alternifolia and iodine in the treatment of molluscum contagiosum in children. J Drugs Dermatol 2012;11:349-54.

66 Aspres N, Freeman S. Predictive testing for irritancy and allergenicity of tea tree oil in normal human subjects. Exogenous Dermatology 2003;2:258-61. 